\title{
Control of Flexible Joint Robotic Manipulator: Design and Prototyping
}

\author{
Waqar Alam ${ }^{1}$, Nihad Ali $^{1}$, Hafiz Muhammad Wahaj Aziz ${ }^{2}$, Jamshed Iqbal ${ }^{3,4, *}$ \\ ${ }^{1}$ Department of Electrical Engineering, COMSATS Institute of Information Technology, Islamabad, Pakistan \\ ${ }^{2}$ Department of Electrical Engineering and Information Technology, Technical University, Munich, Germany \\ ${ }^{3}$ Electrical and Computer Engineering Department, University of Jeddah, Jeddah, Saudi Arabia \\ ${ }^{4}$ Department of Electrical Engineering, FAST National University of Computer and Emerging Sciences, Islamabad, Pakistan \\ *Corresponding author: iqbal.jam@gmail.com
}

\begin{abstract}
Robots are now an integral part of automation sector, thus indicating the importance of the associated control strategies. In contrast with conventional rigid manipulators, flexible arms offer several benefits in terms of light weight and power efficient structure, safe operation due to reduced inertia, low manufacturing cost and faster movements. The present paper proposes a linear control law based on Proportional Integral Derivative (PID) technique to control flexible manipulators. In particular, flexibility in joint is discussed highlighting the control challenges. Tuning of the controller is based on the derived dynamic model using Lagrange approach. The proposed control law is then implemented on a custom-developed platform. Trajectory tracking results witnessed effectiveness of the developed control strategy in terms of reducing undesirable vibrations thereby increasing precision in the flexible manipulator. It is anticipated that the results obtained have enormous potential in applications involving flexible manipulator.
\end{abstract}

Index Terms-Robotics, Flexible robotic arm, Control techniques, Proportional Integral Derivative, Control.

\section{INTRODUCTION}

A robot is defined as an electro-mechanical system, designed to perform specific tasks with speed, accuracy and precision [1]. They come in various shapes and sizes for various tasks and functions. Application areas of robots include but not limited to medical [2,3], space [4], industry [5], nuclear power plants [6], underwater [7] and for target tracking [8]. In medical, they find potential for rehabilitation [9], motion assistance [10,11], cognition [12] etc. The locomotion system of robots is usually based on links [13], legs and wheels [14]. Tremendous research is being carried out in relevant domains including robot modelling and control [15-17]. One very common function performed by modern robots is the manipulation of objects in their surroundings [1]. In general, these manipulators resemble human arms and have multiple Degree of freedom [18].

Robotic manipulators can be further categorized as rigid or flexible. Most robotic manipulators are designed with steel or aluminum frames for increased tensile strength. The result are rigid robotic arms, which are heavy and immobile. With advances in material technology, robotics and the increasing demand of lightweight and portable robotic arms have been the catalyst for research in the design and control of flexible robotic manipulators. Flexibility comes from the use of plastic or carbon-fiber frames, which significantly reduces the cost of manufacturing and the power consumption of the system. But with flexibility come a varying degree of inaccuracies in the robotic arm such as increased settling time, especially at the end point. Fig. 1 illustrates a hardware benchmark with a flexible joint module developed by Quanser.

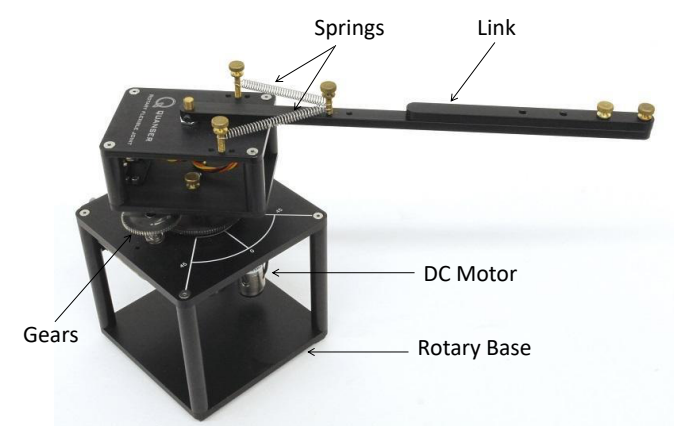

Fig. 1: Flexible joint robotic arm by Quanser [19]

The control algorithms of rigid manipulators are insufficient when it comes to flexible manipulators. Thus special algorithms must be developed to cater for flexibility in the robotic arms and these algorithms must be accurate and compact. An extensive literature review was done into the subject and various linear control strategies available for trajectory tracking control of flexible robot arms have been described. The most commonly used linear control techniques are Proportional Integral Derivative (PID) and Linear Quadratic Regulator (LQR). In [20], PID control law with state feedback control has been proposed for trajectory tracking of flexible joint manipulator. PID control technique can also be hybridized with other linear control techniques to get a more reliable and stable system as well as to improve the overall efficiency and system performance [21].

Another important and very common technique used for linear control of robotic arm systems is the LQR. The reason for the popularity of $L Q R$ is that, in many designs the quadratic cost must be minimized. However in case of tracking the desired trajectory, the response of both LQR and LQR with PID showed similar results [22]. Another advantage of the LQR controller is that, it has a property of phase margin which is greater than 60 and an infinite 
gain margin. However, it cannot deal systematic uncertainties like $\mu$-synthesis and $H \infty$ [23]. Linear approaches because of their simplicity are still the main workhorse in industry even today [24]. The objective of the paper is to design a PID controller for a flexible robotic arm in order to reduce undesirable vibrations at the end-point.

The remaining paper constitutes of following sections: Section II explains the details and categorization of flexible robotic arm, while Section III presents the proposed control design for a flexible robotic arms. Section IV explains the design and fabrication of prototype. The software and hardware simulations are provided in section V. Finally Section VI presents some comments and conclusions.

\section{MAthematicAl Model}

Euler Lagrangian method is utilized to develop the mathematical model for the flexible robot system as shown in Fig.2.

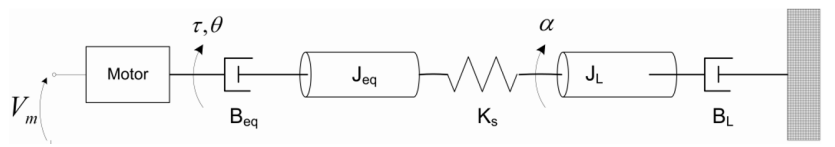

Fig. 2: Schematic model of flexible robotic arm

Various parameters used in modelling are listed in Table 1.

TABLE I: Nomenclature

\begin{tabular}{ccc}
\hline Symbol & Description & Units \\
\hline$I$ & Current flowing inside the motor & $\mathrm{A}$ \\
$V_{m}$ & Voltage applied to the motor & $\mathrm{V}$ \\
$R_{m}$ & Armature resistance & $\Omega$ \\
$k_{m}$ & Motor back EMF constant & $\mathrm{V} . \mathrm{s}$ \\
$k_{g}$ & High gear ratio of motor & - \\
$V_{m}$ & Motor voltage & $\mathrm{V}$ \\
$\tau$ & Motor torque & $\mathrm{N} . \mathrm{m}$ \\
$\theta$ & Angle of DC gear motor & $\mathrm{rad}$ \\
$\alpha$ & Angle of flexible joint & $\mathrm{rad}$ \\
$B_{e q}$ & Viscous fraction coefficient of manipulator & $\mathrm{N} . \mathrm{m} . \mathrm{s} / \mathrm{rad}$ \\
$B_{L}$ & Viscous fraction coefficient of the link & $\mathrm{N} . \mathrm{m} . \mathrm{s} / \mathrm{rad}$ \\
$K_{s}$ & Stiffness of spring & $\mathrm{N} / \mathrm{m}$ \\
$J_{e q}$ & Total moment of inertia of manipulator & $\mathrm{Kgm}$ \\
$J_{L}$ & moment of inertia of the link & $\mathrm{Kgm}$ \\
\hline
\end{tabular}

Lagrange equation deals with the difference of kinetic and the potential energies and is given by

$$
L=T-V
$$

where $T$ and $V$ are rotational kinetic energy and elastic energies respectively and can be expressed as

$$
\begin{aligned}
T & =\frac{1}{2} J \omega^{2} \\
& =\frac{1}{2} J_{e q} \dot{\theta}^{2}+\frac{1}{2} J_{L}(\dot{\theta}+\dot{\alpha})^{2}
\end{aligned}
$$

and

$$
V=\frac{1}{2} K_{s}\left(\alpha^{2}\right)
$$

By substituting (2) and (3), the difference of energies can be rewritten as

$$
L=\frac{1}{2} J_{e q} \dot{\theta}^{2}+\frac{1}{2} J_{L}(\dot{\theta}+\dot{\alpha})^{2}-\frac{1}{2} K_{s}\left(\alpha^{2}\right)
$$

The Euler Lagrange equation is given by

$$
\frac{d}{d t}\left(\frac{\partial L}{\partial q_{i}}\right)-\frac{\partial L}{\partial q_{i}}=Q_{i}
$$

where, $q_{i}=\left[\begin{array}{ll}\theta & \alpha\end{array}\right]^{T}$ and $Q i=\left[\begin{array}{ll}\tau-B_{e q} \dot{\theta} & B_{L} \dot{\alpha}\end{array}\right]$ are generalized coordinates and forces acting on the arm. Taking partial derivative of $\mathrm{L}$ with respect to $Q_{i}$ and putting in (5), we get:

$$
\begin{gathered}
J_{e q} \ddot{\theta}+J_{L}(\ddot{\theta}+\ddot{\alpha})=\tau-B_{e q} \theta \\
J_{L}(\ddot{\theta}+\ddot{\alpha})+K_{s} \alpha=B_{L} \dot{\alpha}
\end{gathered}
$$

Using kirchhoff's laws, the load torque cab be written as

$$
\tau=\frac{k_{g} k_{m} V-k_{m} k_{t} k_{g}^{2} \dot{\theta}}{R_{m}}
$$

By substituting (8), (6) can be written as

$$
J_{e q} \ddot{\theta}+J_{L}(\ddot{\theta}+\ddot{\alpha})=\frac{k_{g} k_{m} V-k_{m} k_{t} k_{g}^{2} \dot{\theta}}{R_{m}}-B_{e q} \theta
$$

(7) and (9) can be a represented in state space form as

$$
\begin{aligned}
\dot{x}_{1} & =x_{2} \\
\dot{x}_{2} & =-\frac{K_{s}}{J_{L}} x_{1}+\frac{B_{L}}{J_{L}} x_{2}+\frac{K_{s}}{J_{L}} x_{3}-\frac{B_{L}}{J_{L}} x_{4} \\
\dot{x}_{3} & =x_{4} \\
\dot{x}_{4} & =-\frac{K_{s}}{J_{e q}} x_{1}+\frac{B_{L}}{J_{e q}} x_{2} \frac{K_{S}}{J_{e q}} x_{3}-\left(\frac{k_{m} k_{t} k_{g}^{2}}{R_{m} J_{e q}}-\frac{B_{L}+B_{e q}}{J_{e q}}\right) x_{4} \\
y & =x_{1}+x_{2}
\end{aligned}
$$

Generally, (10) can be written as

$$
\begin{gathered}
\dot{x}=A x+B u \\
y=C x+D u \\
\text { where } A=\left[\begin{array}{cccc}
0 & 1 & 0 & 0 \\
-\frac{K_{s}}{J_{L}} & \frac{B_{L}}{J_{L}} & \frac{K_{s}}{J_{L}} & -\frac{B_{L}}{J_{L}} \\
0 & 0 & 0 & 1 \\
-\frac{K_{s}}{J_{e q}} & \frac{B_{L}}{J_{e q}} & \frac{K_{s}}{J_{e q}} & \frac{k_{m} k_{t} k_{g}^{2}}{R_{m} J_{e q}}-\frac{B_{L}+B_{e q}}{J_{e q}}
\end{array}\right], \\
B=\left[\begin{array}{c}
0 \\
0 \\
\frac{k_{g} k_{m}}{R_{m} J_{e q}} \\
-\frac{k_{g} k_{m}}{R_{m} J_{e q}}
\end{array}\right], C=\left[\begin{array}{llll}
1 & 1 & 0 & 0
\end{array}\right] \text { and } D=[0]
\end{gathered}
$$

\section{Control Design}

Among the linear control techniques, PID is most commonly used in industry nowadays because of simplicity and ease in control. In the present work, simulations were carried out in MATLAB/Simulink to show the performance of PID controller. The PID-Tuner block tunes the values of the PID constants i.e. $K_{p}, K_{i}$ and $K_{d}$ to find the appropriate values 
which would produce a stable response. Fig. 3 illustrates the block diagram of PID control scheme.

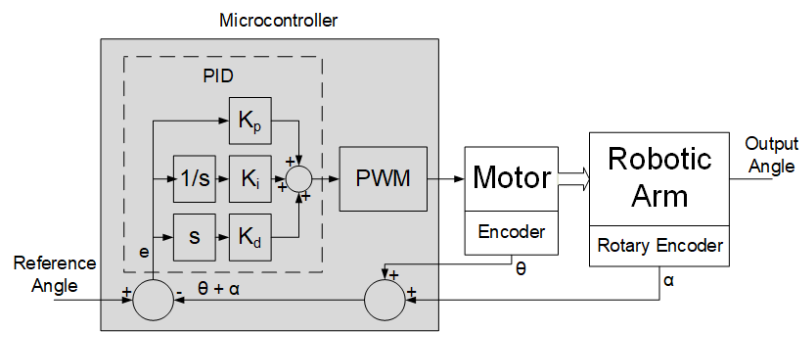

Fig. 3: Block diagram of PID implementation

Tuning a PID controller appears to be conceptually intuitive [25]. To validate the controller gains resulting from simulation, the values of PID gains were then incorporated into the $\mathrm{C}$-code running on Arduino UNO in the real time platform. Algorithm 1 shows pseudo code of implemented PID control law.

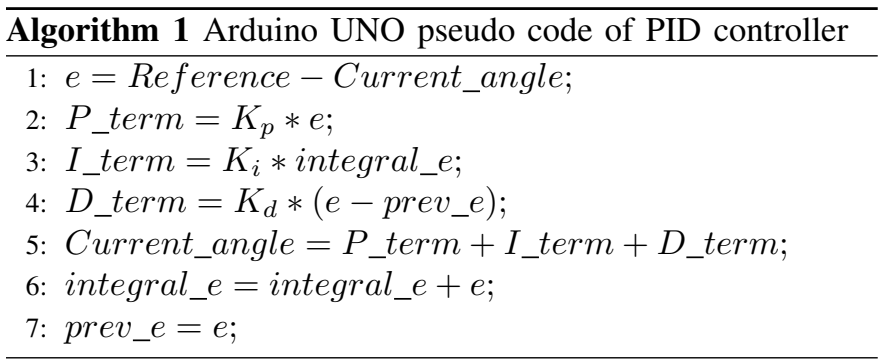

Fig. 4 shows the flow chart of implemented controller code. When the user enters a reference angle as an input, the motor starts to rotate and both encoders, i.e. motor encoder and rotary encoder start returning values. These coded values are mapped using their respective resolutions into angles. The sum of both angles is calculated in order to obtain the current angle. To get the error variable, current angle is subtracted from the

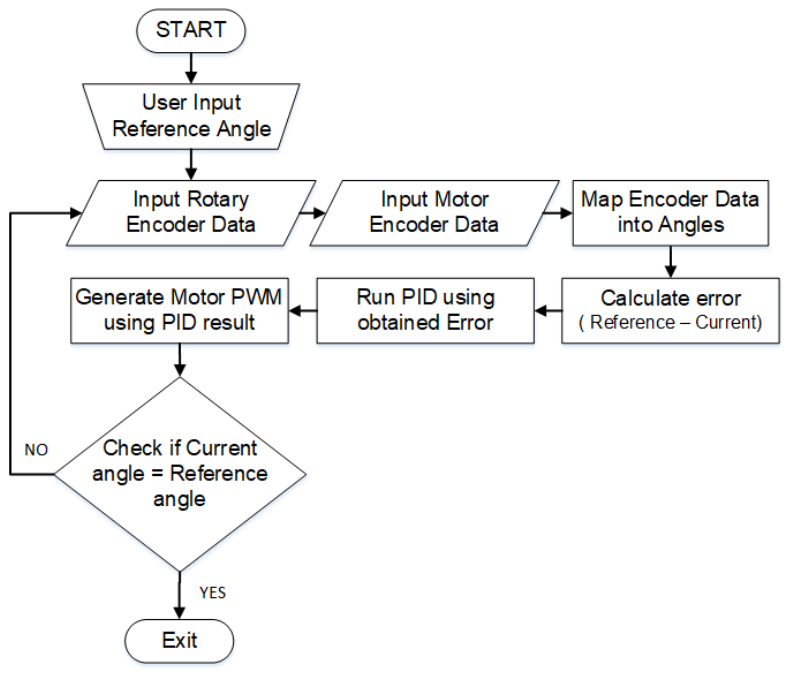

Fig. 4: Flow chart of PID code

reference angle and is given to PID block which implements the pseudo code given in Algorithm 1. The output of this block is passed to a Pulse Width Modulation (PWM) function which controls motor speed. This process continues until the reference angle is achieved where the loop breaks.

\section{DESIGN AND FABRiCATION OF PROTOTYPE}

The simulated control law is then implemented on a customdesigned indigenously developed platform. The first step is to design the CAD model of the flexible robotic arm shown in Fig. 5.

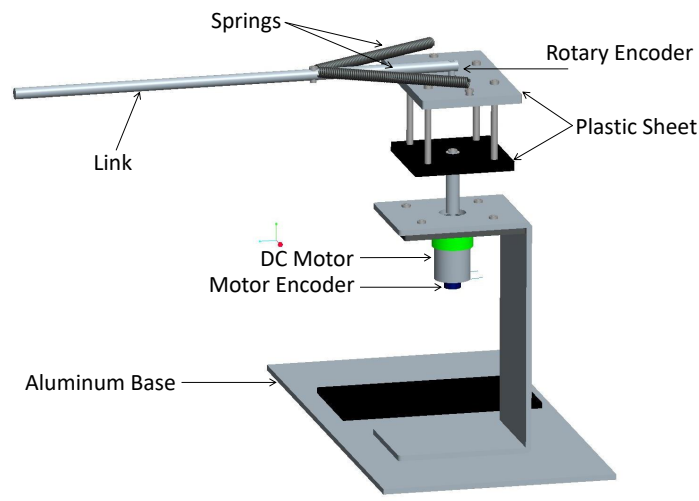

Fig. 5: CAD model of flexible robotic arm

To construct the designed platform, an aluminum sheet was bent at two points to make a base. The one large hole in the center and four in a circle around were drilled. The motor shaft was passed through the central hole and screws were used to mount the motor to the base. A 3 inch aluminum rod was attached vertically, as this small rod will be responsible for keeping the motor attached to the flexible joint rotary base. The rotary base was assembled by taking two square pieces of plastic sheet and passing four long nut-bolts at the corners of both to fashion a two level structure. The upper plate has a three more holes drilled, one in the center and two on the sides. Through the central hole, the rotary encoder shaft was passed towards the top, where it was attached to a $3 \mathrm{ft}$ long aluminum rod acting as the armature. This is the actual joint in the platform. This joint is made flexible by connecting two springs between the aluminum rod and the rotary base. The exploded view and finished assembled platform are presented in Fig. 6.

The electronic components used for making the flexible joint test bench are microcontroller, motor driver IC, brushed DC motor and rotary encoder. The Arduino Uno is a microcontroller board used for this project. It holds the ATmega328P microcontroller. L298N IC is used to drive DC gear motor, which has higher current rating than Arduino can supply. $12 \mathrm{~V}$ brushed DC gear motor is used, which is attached to a gearbox with a gear ratio of 102.083:1. It also has an integrated quadrature encoder that provides a resolution of 64 counts per revolution of the motor shaft. The rotary encoder KY040 is used in prototype, which is an incremental electro-mechanical device that converts the angular position to digital code. 


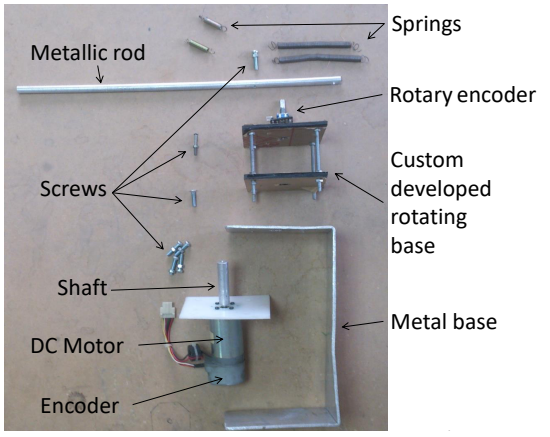

(a)

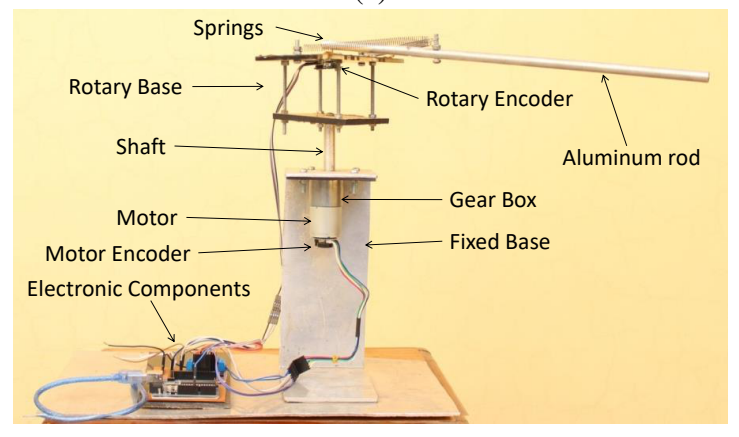

(b)

Fig. 6: Indigenous developed test bench (a) Exploded view (b)Assembled view

During operation, after powering the motor driver IC and Arduino, a reference angle as an input is passed to the Arduino via the USB cable. The Arduino controls the motor through the motor driver IC. As soon as the motor begins to move, there are undesirable deflections due to flexible joint. The deflections are measured using a rotary encoder and are fed back to the Arduino board thus closing the loop.

\section{Results AND Discussion}

To characterize the performance of control law, simulations were performed in MATLAB 2014a, running on a computer system with the following specifications; HP 1000 Notebook PC, Intel(R) Core(TM) i3-2370M CPU @ 2.40GHz, 64-bit Operating System. The designed control law is then implemented on the real platform and responses obtained have been analyzed. The results obtained in both ways supported each other.

In Fig. 7, the simulated output response using step signal as the reference input is illustrated. It is quite clear that the system accurately follows the reference input with a delay of $0.4363 \mathrm{sec}$, without overshooting its reference with large settling time. Fig. 8 demonstrates the tracking performance of the proposed controller when the desired trajectory to be tracked is a sinusoidal signal. It is evident that using the proposed controller the system followed the input waveform and tracking approaches to zero within 4 sec.

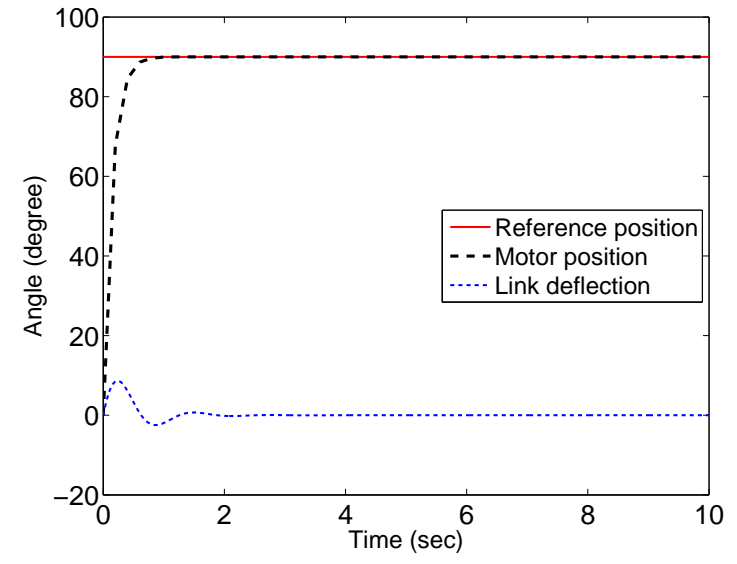

Fig. 7: Simulated trajectory tracking of flexible robotic arm using step input

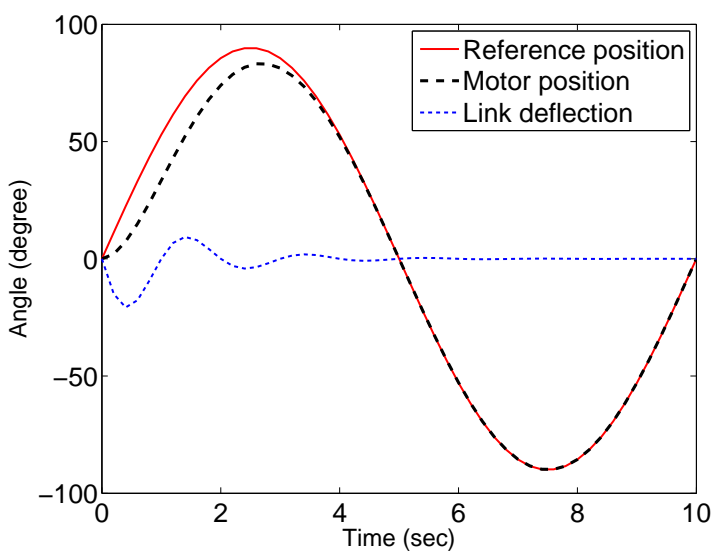

Fig. 8: Simulated trajectory tracking of flexible robotic arm using sinusoidal input

In hardware testing, experiments were conducted on the tracking of sinusoidal waveform and step response using PID control technique. In Fig. 9 experimental result are illustrated where the sinusoidal input is taken as a desired trajectory to be tracked.

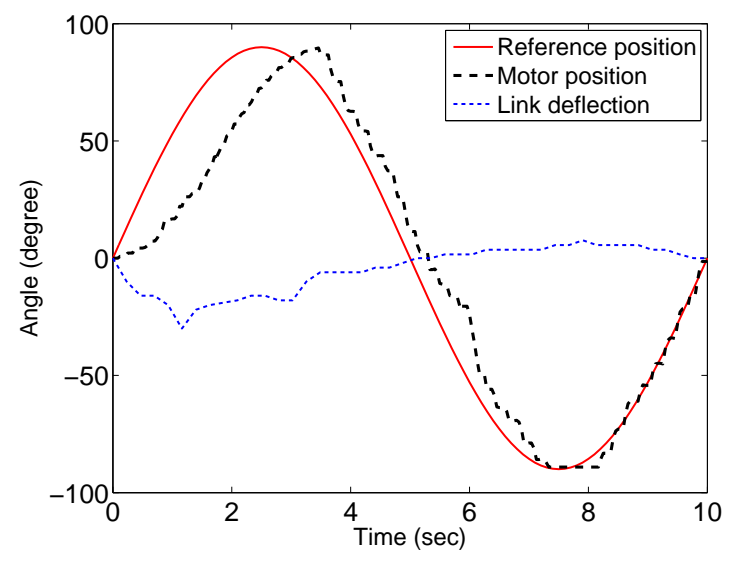

Fig. 9: Experimental sinusoidal trajectory tracking of flexible robotic arm 
The resulting fluctuations measured by the rotary encoder at flexible joint are also depicted. It can be seen that the motor follows the input sinusoidal waveform. The flexible joint angle also shows a major dip in the opposite direction, when the motor angle reaches its peak angle and starts moving in back direction.

Fig. 10 demonstrates the tracking behavior of system for step input as a desired trajectory. It is quite clear that the proposed controller accurately tracks the desired trajectory with better nominal control performance.

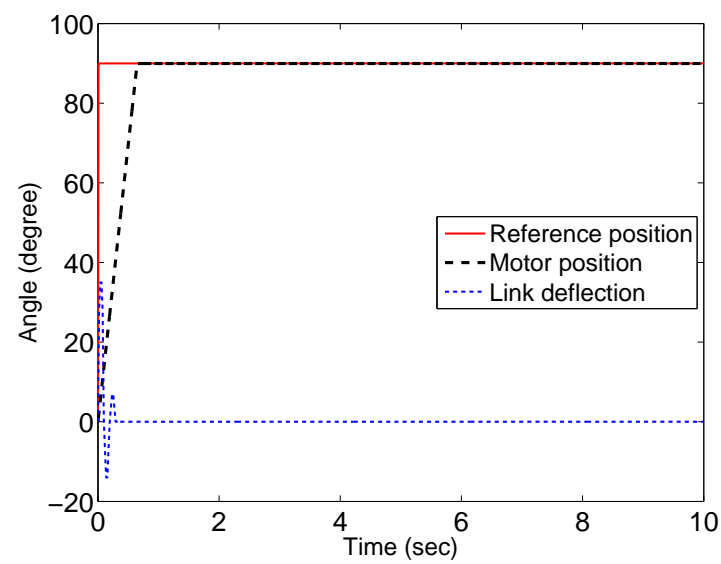

Fig. 10: Experimental step trajectory tracking of flexible robotic arm

To get more and deeper insight the response is plotted for 2 sec. It can be seen in Fig. 11 that there are fluctuations only when the motor starts, which is due to initial jerk to the flexible joint. No fluctuations are detected afterward.

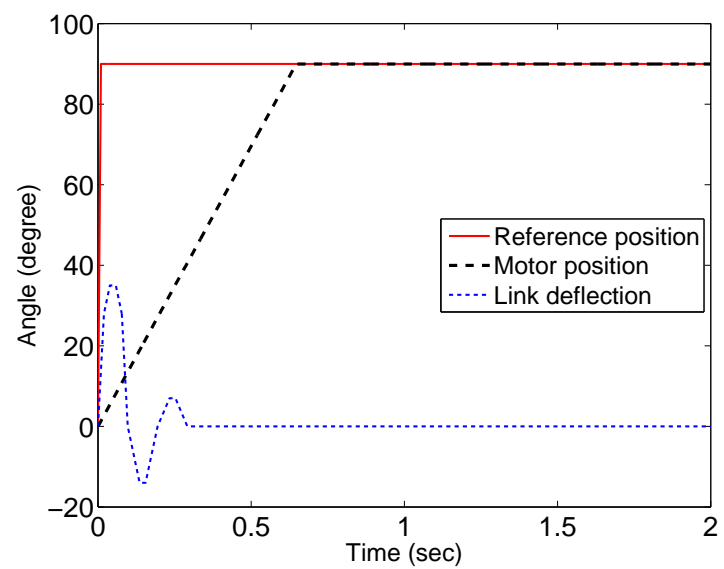

Fig. 11: Zoom view of experimental step reference position tracking

\section{CONCLUSION AND Future WORK}

The first phase of this research was to simulate the vibrations at the end of the flexible robotic manipulator. This was achieved by fixing the armature with the springs which turned it into a flexible joint. After simulating the flexible joint, a PID controller was designed and implemented in order to reduce the vibrations. The vibrations were visibly reduced and results supporting this conclusion were gathered, both from MATLAB/Simulink and from the platform. Consistency in the simulation results and results from real platform validates the effectiveness of the proposed control law. As a future of this work, a nonlinear and robust control scheme may be implemented to further reduce the vibrations in the presence of disturbances.

\section{REFERENCES}

[1] S. A. Ajwad and J. Iqbal, "Emerging trends in roboticsa review from applications perspective," in International Conference on Engineering and Emerging Technologies (ICEET), 2015.

[2] A. A. Khan, S. Riaz, and J. Iqbal, "Surface estimation of a pedestrian walk for outdoor use of power wheelchair based robot," Life Science Journal, vol. 10, no. 3, pp. 1697-1704, 2013.

[3] J. Iqbal and K. Baizid, "Stroke rehabilitation using exoskeleton-based robotic exercisers: Mini review." Biomedical Research, 2015.

[4] J. Iqbal, M. Rehman-Saad, A. Malik, and A. MahmoodTahir, "State estimation technique for a planetary robotic rover," Revista Facultad de Ingeniería Universidad de Antioquia, no. 73, pp. 58-68, 2014.

[5] J. Iqbal, R. U. Islam, S. Z. Abbas, A. A. Khan, and S. A. Ajwad, "Automating industrial tasks through mechatronic systems-A review of robotics in industrial perspective," Tehnički vjesnik, vol. 23, no. 3, pp. 917-924, 2016.

[6] J. Iqbal, A. M. Tahir, R. ul Islam et al., "Robotics for nuclear power plantschallenges and future perspectives," in Applied Robotics for the Power Industry (CARPI), 2012 2nd International Conference on. IEEE, 2012, pp. $151-156$.

[7] A. M. Tahir and J. Iqbal, "Underwater robotic vehicles: Latest development trends and potential challenges." Science International, vol. 26, no. 3, 2014.

[8] J. Iqbal, S. M. Pasha, K. Baizid, A. A. Khan, and J. Iqbal, "Computer vision inspired real-time autonomous moving target detection, tracking and locking," Life Science Journal, vol. 10, no. 4, pp. 3338-3345, 2013.

[9] J. Iqbal, N. Tsagarakis, and D. Caldwell, "Human hand compatible underactuated exoskeleton robotic system," Electronics Letters, vol. 50, no. 7, pp. 494-496, 2014.

[10] J. Iqbal, N. Tsagarakis, and D.Caldwell, "A helping hand," Electron. Lett, vol. 51, p. 880, 2015.

[11] J. Iqbal, N. Tsagarakis, and D. Caldwell, "Four-fingered lightweight exoskeleton robotic device accommodating different hand sizes," Electronics Letters, vol. 51, no. 12, pp. 888-890, 2015.

[12] M. M. Azeem, J. Iqbal, P. Toivanen, and A. Samad, "Emotions in robots," Emerging Trends and Applications in Information Communication Technologies, pp. 144 153, 2012.

[13] S. Manzoor, R. U. Islam, A. Khalid, A. Samad, and 
J. Iqbal, "An open-source multi-dof articulated robotic educational platform for autonomous object manipulation," Robotics and Computer-Integrated Manufacturing, vol. 30, no. 3, pp. 351-362, 2014.

[14] H. Khan, J. Iqbal, K. Baizid, and T. Zielinska, "Longitudinal and lateral slip control of autonomous wheeled mobile robot for trajectory tracking," Frontiers of Information Technology \& Electronic Engineering, vol. 16, no. 2, pp. 166-172, 2015.

[15] S. A. Ajwad, M. I. Ullah, R. U. Islam, and J. Iqbal, "Modeling robotic arms-a review and derivation of screw theory based kinematics," in International Conference on Engineering and Emerging Technologies/Lahore, Pakistan, 2014, p. 98.

[16] S. A. Ajwad, J. Iqbal, M. I. Ullah, and A. Mehmood, "A systematic review of current and emergent manipulator control approaches," Frontiers of Mechanical Engineering, vol. 10, no. 2, pp. 198-210, 2015.

[17] S. A. Ajwad, U. Iqbal, and J. Iqbal, "Hardware realization and pid control of multi-degree of freedom articulated robotic arm," Mehran University Research Journal of Engineering and Technology, vol. 34, pp. 1-12, 2015.

[18] S. Ajwad, M. Ullah, B. Khelifa, and J. Iqbal, "A comprehensive state-of-the-art on control of industrial articulated robots," Journal of Balkan Tribological Association, vol. 20, no. 4, pp. 499-521, 2014.

[19] Quanser. (10-3-2016). Quanser rotary Flexible link. Available:http://www.quanser.com/products/rotary_ flexible_link.

[20] I. H. Akyuz, E. Yolacan, H. M. Ertunc, and Z. Bingul, "Pid and state feedback control of a single-link flexible joint robot manipulator," in Mechatronics (ICM), 2011 IEEE International Conference on. IEEE, 2011, pp. 409-414.

[21] M. Ahmad, M. Suid, M. Ramli, M. Zawawi, and R. R. Ismail, "Pd fuzzy logic with non-collocated pid approach for vibration control of flexible joint manipulator," in Signal Processing and Its Applications (CSPA), 2010 6th International Colloquium on. IEEE, 2010, pp. 1-5.

[22] M. A. Ahmad, "Vibration and input tracking control of flexible manipulator using lqr with non-collocated pid controller," in Computer Modeling and Simulation, 2008. EMS'08. Second UKSIM European Symposium on. IEEE, 2008, pp. 40-45.

[23] C. Olalla, R. Leyva, A. El Aroudi, and I. Queinnec, "Robust lqr control for pwm converters: An lmi approach," IEEE Transactions on industrial electronics, vol. 56, no. 7, pp. 2548-2558, 2009.

[24] C. Chitu, J. Lackner, M. Horn, H. Waser, and M. Kohlbock, "A robust and optimal lqr controller design for electric power steering system," in Nonlinear Dynamics and Synchronization (INDS) \& 16th Int'l Symposium on Theoretical Electrical Engineering (ISTET), 2011 Joint 3rd Int'l Workshop on. IEEE, 2011, pp. 1-5.

[25] K. H. Ang, G. Chong, and Y. Li, "PID control system analysis, design, and technology," IEEE Transactions on
Control Cystems Technology, vol. 13, no. 4, pp. 559-576, 2005. 